\title{
Effect of Next-Generation Exome Sequencing Depth for Discovery of Diagnostic Variants
}

\author{
Kyung Kim ${ }^{1,2,3 \dagger}{ }^{5}$, Moon-Woo Seong ${ }^{4}$, Won-Hyong Chung ${ }^{3}$, Sung Sup Park ${ }^{4}$, Sangseob Leem ${ }^{1}$, \\ Won Park ${ }^{5,6}$, Jihyun Kim ${ }^{1,2}$, KiYoung Lee ${ }^{1,2_{*},{ }^{\ddagger}}$, Rae Woong Park ${ }^{1,2_{*}}$, Namshin Kim ${ }^{5,6 * *}$ \\ ${ }^{1}$ Department of Biomedical Informatics, Ajou University School of Medicine, Suwon 443-749, Korea, \\ ${ }^{2}$ Department of Biomedical Science, Graduate School, Ajou University, Suwon 443-749, Korea, \\ ${ }^{3}$ Korean Bioinformation Center, Korea Research Institute of Bioscience and Biotechnology, Daejeon 305-806, Korea, \\ ${ }^{4}$ Department of Laboratory Medicine, Seoul National University Hospital College of Medicine, Seoul 110-799, Korea, \\ ${ }^{5}$ Department of Functional Genomics, Korea University of Science and Technology, Daejeon 305-806, Korea, ${ }^{6}$ Epigenomics \\ Research Center, Genome Institute, Korea Research Institute of Bioscience and Biotechnology, Daejeon 305-806, Korea
}

Sequencing depth, which is directly related to the cost and time required for the generation, processing, and maintenance of next-generation sequencing data, is an important factor in the practical utilization of such data in clinical fields. Unfortunately, identifying an exome sequencing depth adequate for clinical use is a challenge that has not been addressed extensively. Here, we investigate the effect of exome sequencing depth on the discovery of sequence variants for clinical use. Toward this, we sequenced ten germ-line blood samples from breast cancer patients on the Illumina platform GAll(x) at a high depth of $\sim 200 \times$. We observed that most function-related diverse variants in the human exonic regions could be detected at a sequencing depth of $120 \times$. Furthermore, investigation using a diagnostic gene set showed that the number of clinical variants identified using exome sequencing reached a plateau at an average sequencing depth of about $120 \times$. Moreover, the phenomena were consistent across the breast cancer samples.

Keywords: clinical application, diagnostic variant, exome sequencing, genetic variation, high-throughput nucleotide sequence variant, sequencing

\section{Introduction}

Exome capture sequencing (simply referred to as "exome sequencing") is a next generation sequencing (NGS)-based technique which targets the genomic sequences of protein-coding regions ("exomes") of a species [1]. Although protein-coding regions constitute only $1 \%$ of the human genome, they harbor $85 \%$ of the mutations that have significant effects on disease-related traits [2]. Therefore, exome sequencing is a potential contributor to the understanding of diverse human diseases [2].

With a dramatic decrease in the cost and time required for the generation of sequences with high accuracy [3], exome sequencing is now widely used to understand many genetic diseases. For example, in the Netherlands, exome sequencing of ten blood samples from patients with severe intellectual disabilities allowed the identification of five new candidate genes associated with such disabilities [4]. Further, Ng et al. [1] sequenced the exomes of twelve human samples with or without Freeman-Sheldon syndrome (FSS), which is a rare dominantly inherited disorder, and observed an association between the MYH3 gene was responsible for FSS. Furthermore, Huh et al. [5] used exome sequencing to show that the c.234 $\mathrm{G}>\mathrm{A}$ and c.1150C $>\mathrm{T}$ mutations in exon 18 of the HGSNAT gene were common in mucopolysaccharide patients. Exome sequencing techniques have also been used to understand the risks of various cancers, including those of

Received April 8, 2015; Revised May 26, 2015; Accepted May 28, 2015

*Corresponding author 1: Tel: +82-31-219-4471, Fax: +82-31-219-4472, E-mail: veritas@ajou.ac.kr

**Corresponding author 2: Tel: +82-42-879-8162, Fax: +82-42-879-8493, E-mail: n@rna.kr

${ }^{\dagger}$ These two authors contributed equally to this work.

${ }^{\ddagger}$ Deceased.

Copyright (C) 2015 by the Korea Genome Organization

(c) It is identical to the Creative Commons Attribution Non-Commercial License (http://creativecommons.org/licenses/by-nc/4.0/). 
the breast [6, 7], prostate [8], pancreas [9], and others [10-12]. Therefore, exome sequencing techniques have become a new primary paradigm for research on genetic diseases and cancers.

One important issue that needs to be addressed for the clinical utilization of NGS-based sequencing data is the adequate depth of sequencing. Sequencing depth is directly related to the cost and time required for the generation, processing, and maintenance of sequencing data [13]. In this vein, several studies have been performed to investigate the impact of sequencing depth on NGS data intended to identify genomic variants. For example, Hou et al. [14] investigated the discovery rates of single nucleotide polymorphisms (SNPs) and structural variants in healthy samples using whole genome sequencing (WGS) at an average sequencing depth of 180x. They observed that most of the variations were identified at an average depth of 100x. Meanwhile, Ajay et al. [15] calculated the genome coverage and discovery rates of variants in healthy samples using WGS data at 100x average mapped depth. The callable portion of the genome was $90 \%$ at a depth of $40 \times$, and that of the protein-coding exome region was about $88 \%$ at a depth of $100 \times$. However, these studies analyzed sequencing data derived from healthy individuals; the adequate depth required to discover clinically significant variations still needs to be addressed.

In this study, we investigated for the first time the effect of exome sequencing depth on the discovery of genomic variations for clinical use. Toward this, we performed exome sequencing in ten germ-line blood samples from breast cancer patients using the Illumina platform GAII(x) at a high depth of $\sim 200 \times$. We also checked the discovery rates of diverse variations as a function of the sequencing depth, using total and diagnostic gene sets.

\section{Methods}

\section{Samples and sequencing}

Total ten subjects were included in this study. They were enrolled from the Seoul National University Hospital and Seoul National University Bundang Hospital in Korea. They were all diagnosed with breast cancer, with a history of two or more affected family members and/or other risk factors, like bilateral breast cancer or young age of onset. Exome capture was carried out with the blood samples of the ten subjects using Agilent exome capture kits (SureSelect V2) and sequencing was performed on the (Illumina, San Diego, CA, USA).

\section{Sequence alignment and variant calling}

The raw reads in the prepared datasets were aligned to the hg19 reference genome, which was downloaded from the
University of California Santa Cruz (UCSC) genome browser (http://genome.ucsc.edu/), using BWA (bwa-0.6.2) [16] with default parameters and a seed length of $45 \mathrm{bp}$. The Sequence Alignment and Mapping (SAM) files were converted to Binary Alignment and Mapping (BAM) files using SAMtools [17]. Picard (http://picard.sourceforge.net/) was used to mark and remove the polymerase chain reaction duplicates detected from the BAM files. The Genome Analysis Toolkit (GATK) [18] was then used for base quality recalibration and local realignment around the potential indel sites. The UnifiedGenotyper [19] in the GATK was used in the final step for variant calling using a Bayesian model. Variants were filtered by three types of filtering methods: (1) HARD_TO_VALIDATE: MQO $\geq 4$ and [(MQO/(1.0 × DP) $]>0.1$; (2) QualFilter: QUAL < 10; and (3) Additional: QUAL $<30.0|| \mathrm{MQ}<20.0|| \mathrm{DP}<7$.

\section{Annotation of genetic variants}

We annotated variants using diverse tools and databases. The region information of variants (such as coding or intron regions and splice sites) was annotated using SnpEff [20]. Further, we predicted the functional effects of variations on genes (such as silent, nonsense, or missense SNPs) using SnpEff [20]. We also predicted whether an amino acid substitution significantly affects protein function (such as deleterious or tolerated SNPs) using SIFT [21]. In addition, we checked previously known SNPs using the dbSNP database [22]. We also checked clinical SNPs using the ClinVar database [23].

\section{Analysis of depth of coverage in the diagnostic gene set}

We extracted information for the positions of the 175 diagnostic genes from the hg19 reference genome, which was downloaded from the UCSC genome [24]. Based on this, we analyzed the depth of coverage and mapped mean depth according to increasing sequencing depths using Samtools "mpileup" with default parameters [17].

\section{Count analysis of diverse variants}

We calculated counts of the number of diverse clinical variants including nonsense, missense, and deleterious SNPs in coding regions of the total genome and diagnostic genes using in-house scripts.

\section{Results and Discussion}

\section{Effect of exome sequencing depth on the discovery of variants for clinical use}

To investigate the effect of exome sequencing depth on the discovery of clinically meaningful variants, we first sequenced 
the whole blood DNA samples from ten breast cancer patients using the Illumina platform GAII(x) at a high sequencing depth of $\sim 200 \times$ (Fig. 1A). The platform covers $95 \%$ of the human exonic regions (http://www.gemomics. agilent.com). We then extracted data at depths of $20 \times$ to $200 \times$ by random selection and shuffling (Fig. 1B). Next, we performed read mapping, local realignment, duplicate marking, and base quality recalibration for each sample (Fig. 1C). Diverse variations such as SNPs, and insertions and deletions (indels) were called by using the GATK [16]. We also called function-related variations after the annotation of functional and regional information using various open databases and tools (Methods). Finally, we analyzed counts, positions, reading depths, and genomic coverage of the identified variations as a function of the sequencing depth using total or diagnostic gene sets (Fig. 1D).

We first checked the numbers of called SNPs in protein-coding (denoted as "coding SNPs") and total genomic regions (denoted as "total SNPs") of the platform in order to assess the effect of sequencing depth on the discovery of SNPs (Fig. 2A). As expected, the numbers of the identified total SNPs increased at higher average sequencing depths. For example, the median number of total SNPs for the ten samples gradually increased from 33,765 at $20 \times$ to 114,707 at $200 \times$ (an increase of 80,942 for the total SNPs). However, the median number of SNPs in the coding regions increased more rapidly in the first half (an increase of 59,073 from 20x to $120 \times$ ), and reached a plateau at an average sequencing depth of around $120 \times$. The increase in the number of coding SNPs was significantly smaller after that (an increase of 827 from $120 \times$ to $200 \times$ ). This also means that the number of non-coding SNPs detected, both intronic and intergenic, increased rapidly after a depth of $120 \times$ was achieved. This phenomenon was more or less consistent across the sequencing data, from the first sample to the last. This trend was also preserved in the indel variants (Supplementary Fig. 1). Further, the number of indels in the total region of the platform (denoted as "total indels") increased steadily, but the increasing ratio of indels in the coding regions ("coding indels") leveled off after a depth of $120 \times$ was achieved.

After functional annotation using the SnpEff database [20], we analyzed the numbers of functional SNPs (nonsense,

\section{(A) Exome sequencing ( 200x depth for each sample using Illumina GAII(x))}

(B)

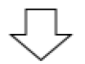

20x - 200x extractions by random selections and random shuffling (three times)

(C) $)_{\text {Read mapping and variant calling with quality control using different depths }}$

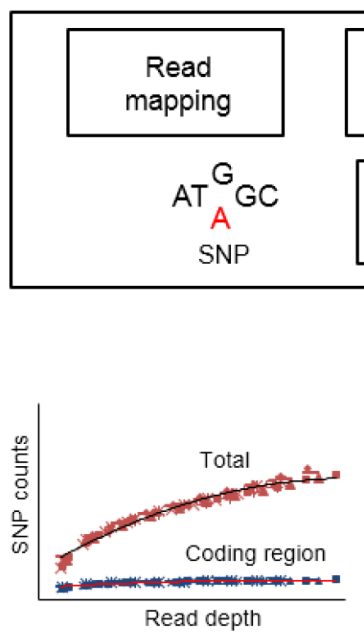

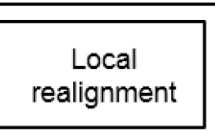

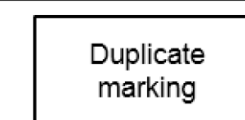

Variant calling and annotation

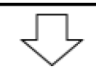

(D)

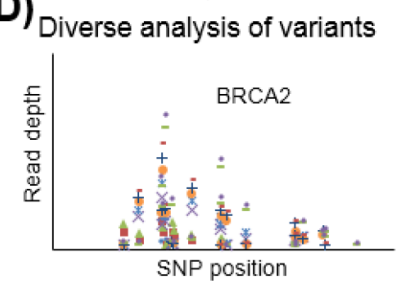

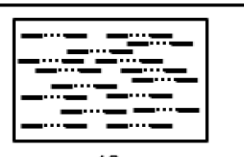

Sample 10
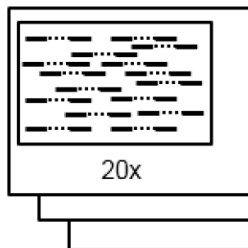

$40 \mathrm{x}$

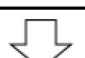

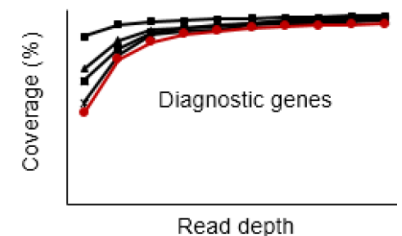

Fig. 1. Study overview. (A) Whole exome deep sequencing of whole blood DNA samples from ten breast cancer patients at $\sim 200 \times$ depth using the Illumina GAll(x). (B) Generation of ten datasets from $20 \times$ to $200 \times$ depths. Each dataset was selected independent of the others. (C) Read mapping and variant calling with quality control. Variations such as indels and single nucleotide polymorphisms (SNPs) were annotated with related functional and regional information. (D) Various analyses of identified variants, such as number of variants, read depth, and genomic coverage according to the mapped depths using both the whole genome and diagnostic genes. 

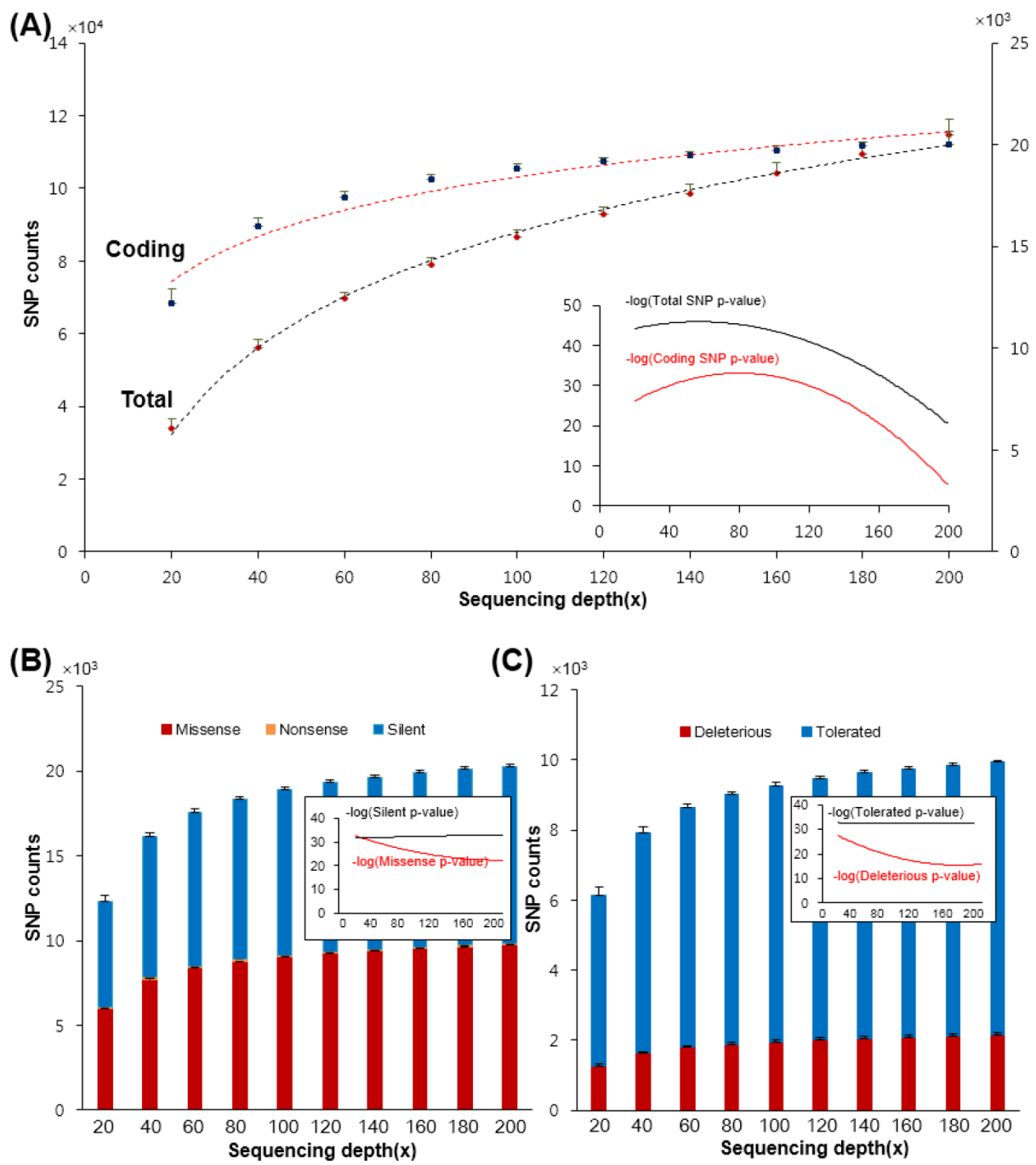

(C) $\times 10$

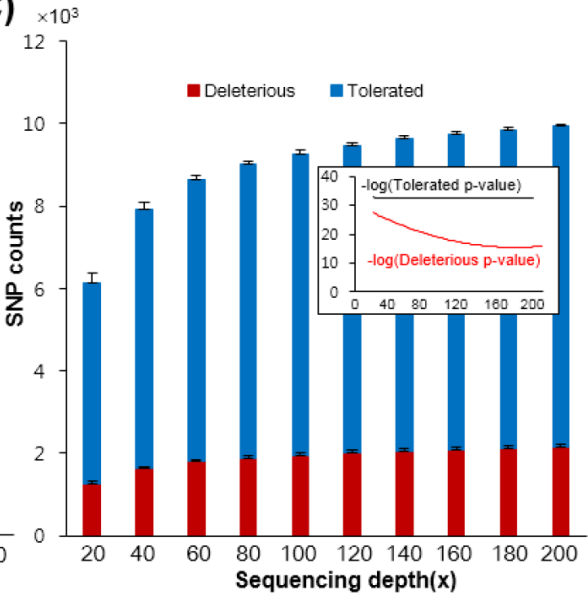

Fig. 2. Numbers of called functional single nucleotide polymorphisms of human genes according to sequencing depths. (A) Numbers of called coding (blue) and total single nucleotide polymorphisms (SNPs) (red) with different sequencing depths: dashed lines are trend lines of means and their error ranges are represented. Solid lines in the inner chart represents how numbers of SNPs are significantly different from each own preceding. (B) The bar chart illustrates the numbers of called silent (blue), nonsense (orange), and missense (red) SNPs. (C) The bar chart illustrates the numbers of called tolerated (blue) and deleterious (red) SNPs in the SIFT database. (B) and (C) represent median values of the ten samples. missense, and silent) while increasing the sequencing depth. The results indicate that the median number of missense SNPs detected in the ten samples increased from 6,015 to 9,731 (Fig. 2B). However, the rate of increase was sharp in the first half, slowing significantly after $120 \times$. We observed a similar trend for the nonsense SNPs as well. In addition, this phenomenon was also observed for the deleterious SNPs using the SIFT [21] (Fig. 2C). In summary, the number of deleterious SNPs detected, such as missense and nonsense, increased with the sequencing depth, but the ratio of this increase reduced significantly after $120 \times$.

Mutations in splice sites are associated with many diseases [25-27]. Therefore, we next analyzed the number of SNPs detected near splice sites with respect to the sequence depth (Fig. 3). We found that the number of called SNPs increased with increasing depth. However, many of the SNPs were detected in intronic regions around the splice sites, about twice the number of those detected in exonic regions, when increasing the sequencing depth. The number of detected SNPs, though, was lowest at the splice sites. This might imply that the sequences of the exonic regions surrounding the splice sites are well conserved than those of the intronic regions. However, researchers who wish to detect susceptible SNPs in splice sites might have to sequence at depths of more than $120 \times$. Similarly, all the variations reported in the dbSNP database were also more common in the intronic, rather than the exonic regions (Supplementary Fig. 2).

To summarize, the number of deleterious SNPs and indels detected in the coding regions (which are widely used in clinical diagnostics) was only weakly increased a depths more than $120 \times$. In other words, a sequencing depth of $120 \times$ can be considered reasonable when using the exome capture sequencing technique to identify significant variations in diagnostic studies.

\section{Exome sequencing depth for diagnostic genes}

Next, we analyzed the genomic coverage and mean depths 

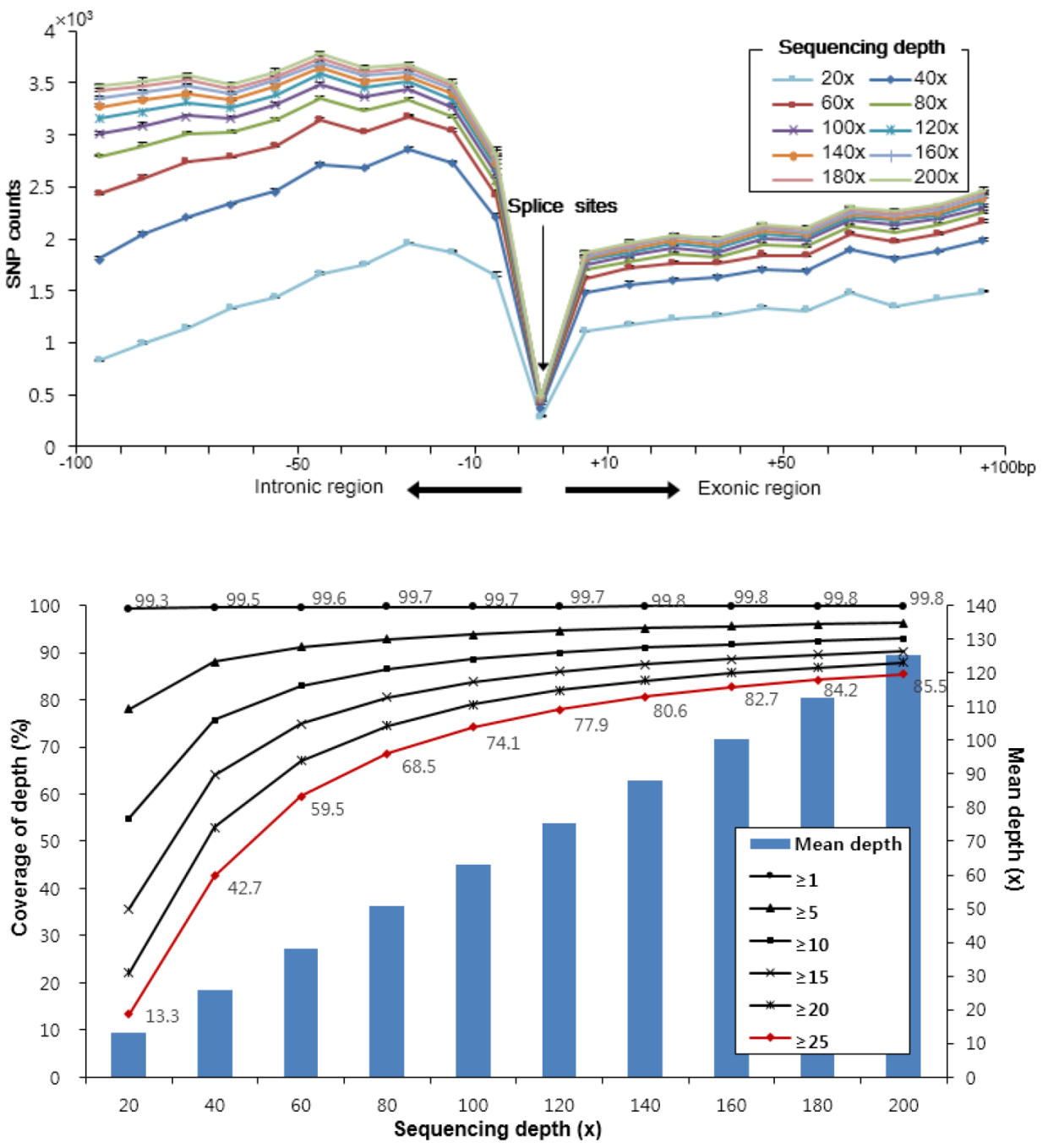

Fig. 3. Numbers of single nucleotide polymorphisms (SNPs) near splice sites. Median numbers of SNPs for the ten samples are depicted. Different colors indicate increasing sequencing depths.

Fig. 4. Mean mapped depth and coverage of diagnostic genomic regions according to sequencing depth. Lines indicate the coverage proportion of the genomic regions of 196 diagnostic genes with specific minimum depths, according to the sequencing depths. Red line: genomic coverage $>25 \times$. Bars indicate mean mapped depths (mean depth).

for 175 diagnostic genes (Supplementary Table 1) with respect to the diverse depths used. These genes have been widely used for diagnostic, prognostic, and therapeutic purposes at the Seoul National University Hospital in Korea. Specifically, these diagnostic genes are a subset of genes from the exome dataset. The percentage of mapped sequences in the coding regions of the 175 genes was almost constant for each depth in the individual samples, although there were slight variations across the samples (Supplementary Table 2). We further observed that the distributions of the average mapped depths between all human genes in the platform and the diagnostic genes were similar (Supplementary Fig. 3). Moreover, with increasing depths, as expected, the median average mean depth of the ten samples increased almost constantly from a depth of $13.3 \times$ to $125.1 \times$ (on average, an increase of $12.4 \times$ per depth) (bar charts in Fig. 4). In contrast, the coverage curves for the coding regions of the diagnostic genes followed logarithmic trends, regardless of the thresholds of the minimum read depths (line charts in Fig. 4). For example, based on the regions with more than $1 \times$ mapped depth, the coverage increased from $99.3 \%$ to $99.7 \%$ (0.4\% increase) at $120 \times$ depth compared to that at $20 \times$. However, the coverage increased by only $0.1 \%$ at a depth of $80 \times$ (totally, $200 \times$ ). Similarly, at $25 \times$ minimum mapped depth, over $77.9 \%$ of the genomic regions were covered when $120 \times$ was used. Moreover, a $64.6 \%$ increase in coverage was observed in the first half (from $13.3 \%$ at $20 \times$ to $77.9 \%$ at $120 \times$ ), whereas a $7.6 \%$ increase was observed in the latter (from $77.9 \%$ at $120 \times$ to $85.5 \%$ to at $200 \times$ ). It is known that a $25 \times$ mapping depth is the minimum for detecting heterozygous alleles $[28,29]$. In other words, the genomic coverage of the diagnostic genes was not increased significantly after $120 \times$.

We next checked the number and positions of SNPs that have been detected in the early onset breast cancer 2 gene (BRCA2), one of the major risk factors in the development of this cancer $[30,31]$. The results indicate that the read depths of SNPs in BRCA2 increased with increasing sequencing 

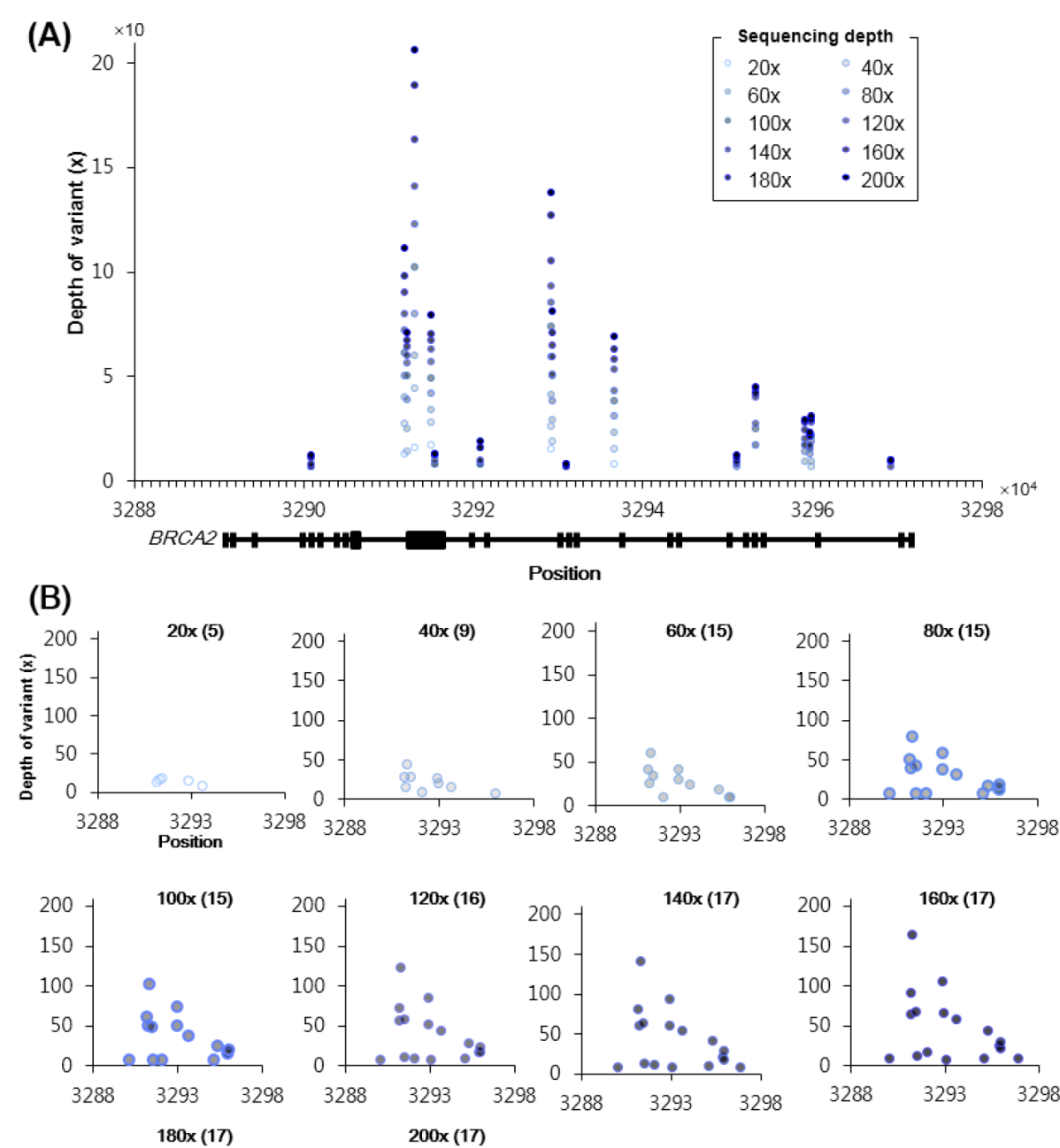

Fig. 5. Mapped depths and positions of the called single nucleotide polymorphisms (SNPs) in BRCA2. (A) Mapped depths (y-axis) of called coding SNPs in BRCA2 (x-axis) according to increasing sequencing depths. The figure at the bottom indicates the exonic regions of $B R C A 2$. (B) Different views of panel (A) based on increasing sequencing depths. The number of called SNPs is presented in parenthesis. For example, $120 \times(16)$ indicates that 16 SNPs were successfully called in the $120 \times$ dataset.

depths (Fig. 5A). However, most of the SNPs had been identified by the time $120 \times$ depth was reached, and no more were identified after $140 \times$ depth was used (Fig. 5B). Only mapped read depths increased with increasing sequence depths. Similar phenomena were also observed in the early onset breast cancer 1 gene (BRCA1) (Supplementary Fig. 4), another major risk factor in this cancer type [32].

Further, we validated our depth recommendation for diagnostic variant detection using exome sequencing. To this end, we analyzed the numbers of non-synonymous (NS) SNPs, splice site acceptor or donor site (SS), and coding indel (I) variants in the diagnostic genes as a function of the diverse depths used (Fig. 6). The numbers of NS/SS/I variants of all human genes in the platform increased with increasing sequencing depths (Fig. 6A). However, the numbers of NS variants in the diagnostic genes converged sooner; there was least increase in the NS variants after $60 \times$ was used (Fig. 6B). We analyzed the numbers of NS variants using the variations in the ClinVar database [23], which is well known for clinical variants. Although there was an increase in the numbers of NS clinical variants, the rate of increase was quite low after $120 \times$ was used (Fig. 6C). We also checked the variations related to breast cancer using only the clinical variants in the ClinVar database (Fig. 6D). The results indicate that all variations associated with breast cancer were detected at $140 \times$ depth, regardless of the sample. In summary, with exome capture sequencing technique, the most significant clinical variations can be detected at an average depth of $120 \times$.

Finally, we measured the discovery rates of variants at 
(A)

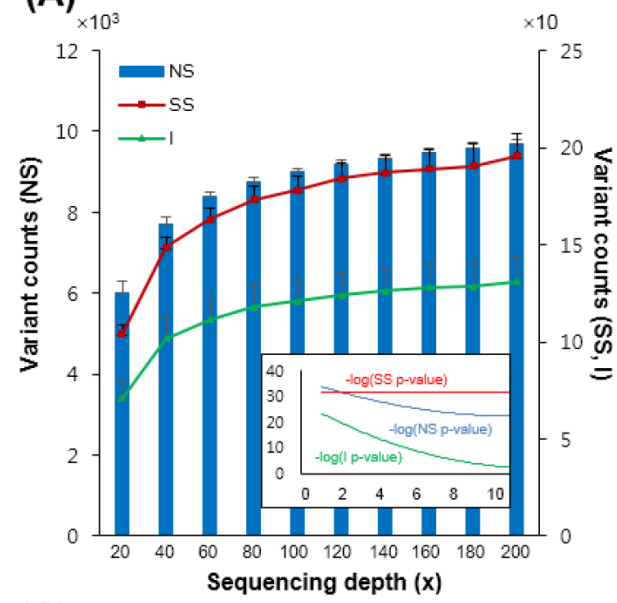

(C)

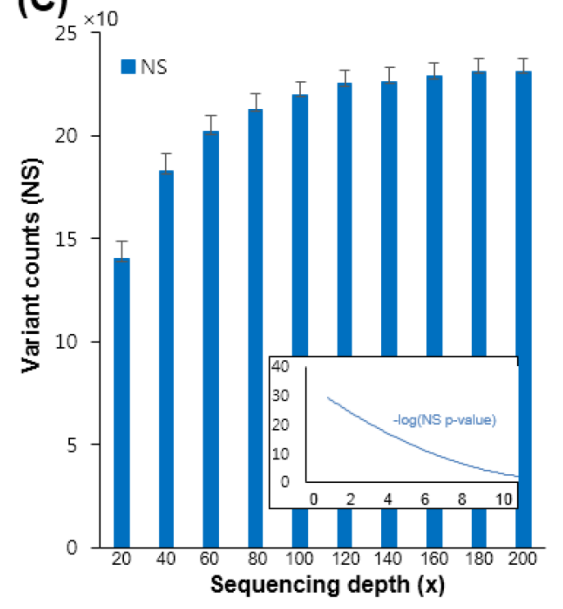

(B)

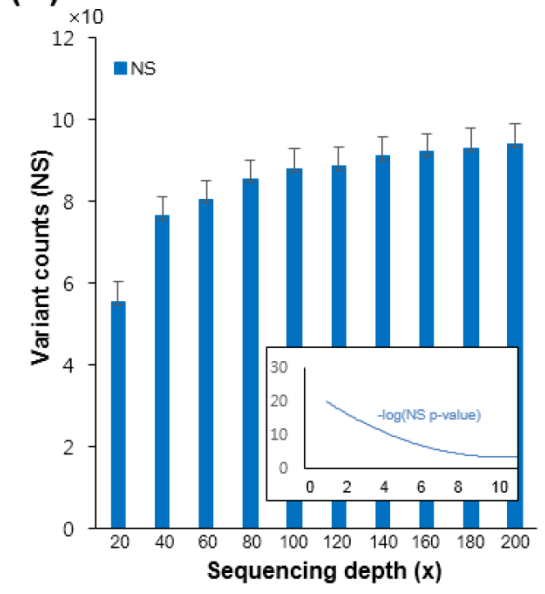

(D)

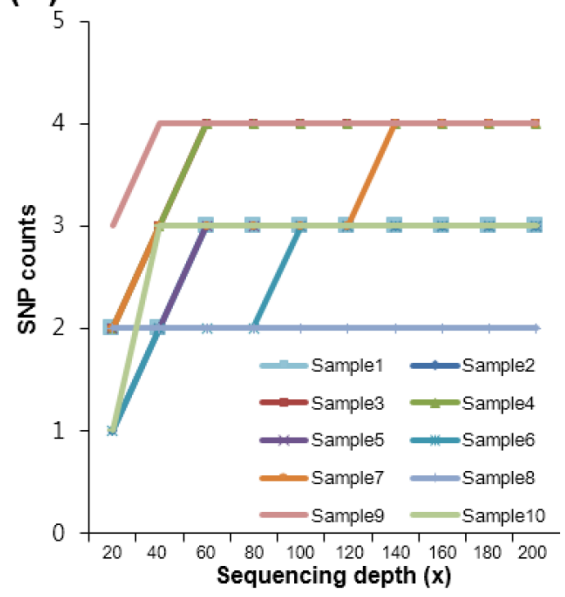

Fig. 6. Numbers of called non-synonymous (NS)/splice site acceptor or donor site (SS)/coding indel (I) in the diagnostic gene set and the ClinVar database according to different sequencing depths. (A, B) Numbers of $\mathrm{NS} / \mathrm{SS} / \mathrm{I}$ per depth used in the human genome (A) or in the 175 diagnostic genes (B). (C) Numbers of NS for the diagnostic genes in the ClinVar database per number of depth used. (D) Numbers of variants in the genes associated with breast cancer among the diagnostic genes in the ClinVar database. The $x$-axis represents increasing sequencing depths.

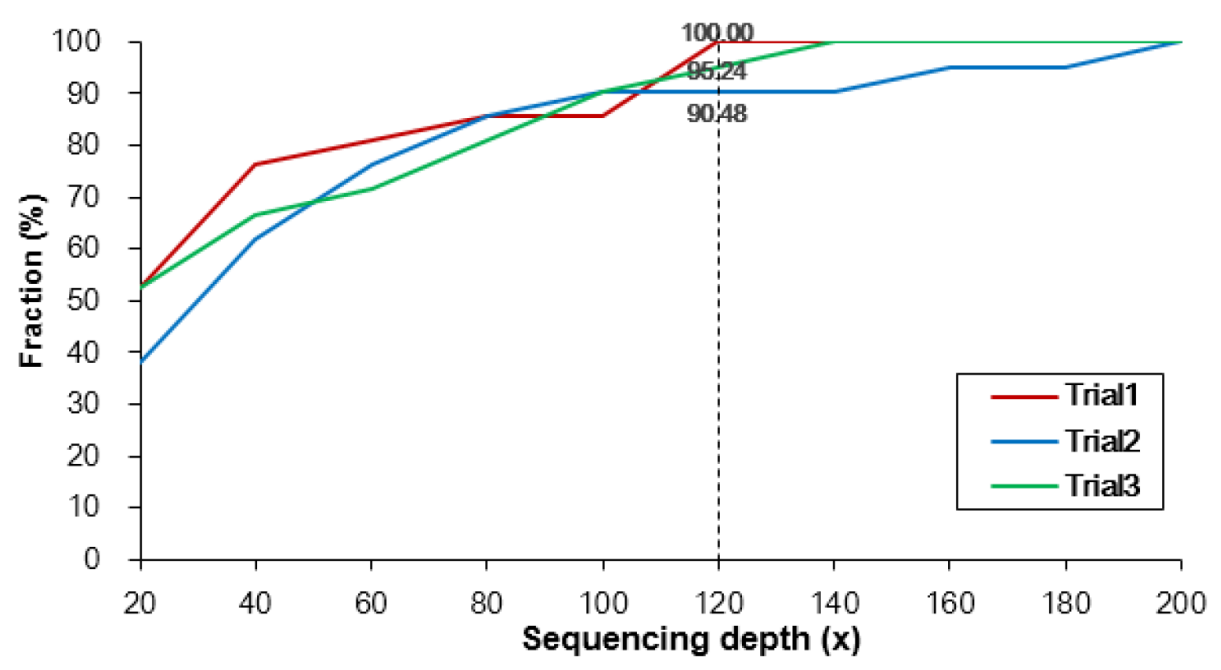

Fig. 7. Fraction of 21 validated single nucleotide polymorphisms (SNPs) in the called SNP set according to different sequencing depths. The median numbers of called SNPs are depicted for the ten samples. Different colors indicate independent trials. different sequencing depths using Sanger sequencing (Fig. 7). The results show that more than $90 \%$ variations were validated when $120 \times$. Hence, we conclude that, using the exome capture sequencing technique, the most reliable variants are detected at an average depth of $120 \times$.

In this study, we determined the effects of exome sequencing depth on the discovery of function-related diverse variants of human genes and diagnostic genes, 
especially for clinical use. For this purpose, we investigated the exome deep-sequencing data for whole blood DNA samples obtained from ten breast cancer patients using an Illumina platform GAII(x) as a function of sequencing depth.

The number of genomic variants identified using exome sequencing reached a plateau at an average sequencing depth of $\sim 120 \times$, and this depth allowed detection of most variations in the human genes. The results were also consistent with a diagnostic gene set and were similar across samples. Considering the diverse costs and time related to generation, processing, and maintenance of sequencing data, this suggests that a feasible depth for clinically relevant exome sequencing is about $120 \times$. These findings can be used to address important questions on the adequate depth for exome sequencing techniques for clinical use.

\section{Supplementary materials}

Supplementary data including two tables and four figures can be found with this article online at http//www. genominfo.org/src/sm/gni-13-31-s001.pdf.

\section{Acknowledgments}

This research was supported by a grant from the Korea Health Technology R\&D Project through the Korea Health Industry Development Institute (KHIDI), funded by the Ministry of Health and Welfare, Republic of Korea (grant number: HI14C32010100). It was also supported by grants from the National Research Foundation of Korea (NRF2011-0030049, NRF-2014M3C9A3064552), Next-Gen BioGreen21 (PJ008019, PJ008068), and the KRIBB initiative program.

\section{References}

1. Ng SB, Turner EH, Robertson PD, Flygare SD, Bigham AW, Lee $\mathrm{C}$, et al. Targeted capture and massively parallel sequencing of 12 human exomes. Nature 2009;461:272-276.

2. Choi M, Scholl UI, Ji W, Liu T, Tikhonova IR, Zumbo P, et al. Genetic diagnosis by whole exome capture and massively parallel DNA sequencing. Proc Natl Acad Sci U S A 2009; 106:19096-19101.

3. Gullapalli RR, Desai KV, Santana-Santos L, Kant JA, Becich MJ. Next generation sequencing in clinical medicine: Challenges and lessons for pathology and biomedical informatics. J Pathol Inform 2012;3:40.

4. de Ligt J, Willemsen MH, van Bon BW, Kleefstra T, Yntema HG, Kroes T, et al. Diagnostic exome sequencing in persons with severe intellectual disability. N Engl J Med 2012; 367:1921-1929.

5. Huh HJ, Seo JY, Cho SY, Ki CS, Lee SY, Kim JW, et al. The first
Korean case of mucopolysaccharidosis IIIC (Sanfilippo syndrome type C) confirmed by biochemical and molecular investigation. Ann Lab Med 2013;33:75-79.

6. Thompson ER, Doyle MA, Ryland GL, Rowley SM, Choong DY, Tothill RW, et al. Exome sequencing identifies rare deleterious mutations in DNA repair genes FANCC and BLM as potential breast cancer susceptibility alleles. PLoS Genet 2012; 8:e1002894.

7. Park DJ, Odefrey FA, Hammet F, Giles GG, Baglietto L, ABCFS, et al. FAN1 variants identified in multiple-case early-onset breast cancer families via exome sequencing: no evidence for association with risk for breast cancer. Breast Cancer Res Treat 2011;130:1043-1049.

8. Lonigro RJ, Grasso CS, Robinson DR, Jing X, Wu YM, Cao X, et al. Detection of somatic copy number alterations in cancer using targeted exome capture sequencing. Neoplasia 2011; 13:1019-1025.

9. Wang L, Tsutsumi S, Kawaguchi T, Nagasaki K, Tatsuno K, Yamamoto $\mathrm{S}$, et al. Whole-exome sequencing of human pancreatic cancers and characterization of genomic instability caused by MLH1 haploinsufficiency and complete deficiency. Genome Res 2012;22:208-219.

10. Le Gallo M, O'Hara AJ, Rudd ML, Urick ME, Hansen NF, $\mathrm{O}$ 'Neil NJ, et al. Exome sequencing of serous endometrial tumors identifies recurrent somatic mutations in chromatin-remodeling and ubiquitin ligase complex genes. Nat Genet 2012;44:1310-1315.

11. Wang K, Kan J, Yuen ST, Shi ST, Chu KM, Law S, et al. Exome sequencing identifies frequent mutation of ARID1A in molecular subtypes of gastric cancer. Nat Genet 2011;43:1219-1223.

12. Liu P, Morrison C, Wang L, Xiong D, Vedell P, Cui P, et al. Identification of somatic mutations in non-small cell lung carcinomas using whole-exome sequencing. Carcinogenesis 2012;33:1270-1276.

13. Cao CC, Li C, Huang Z, Ma X, Sun X. Identifying rare variants with optimal depth of coverage and cost-effective overlapping pool sequencing. Genet Epidemiol 2013;37:820-830.

14. Hou R, Yang Z, Li M, Xiao H. Impact of the next-generation sequencing data depth on various biological result inferences. Sci China Life Sci 2013;56:104-109.

15. Ajay SS, Parker SC, Abaan HO, Fajardo KV, Margulies EH. Accurate and comprehensive sequencing of personal genomes. Genome Res 2011;21:1498-1505.

16. Li H, Durbin R. Fast and accurate long-read alignment with Burrows-Wheeler transform. Bioinformatics 2010;26:589-595.

17. Li H, Handsaker B, Wysoker A, Fennell T, Ruan J, Homer N, et al. The sequence alignment/Map format and SAMtools. Bioinformatics 2009;25:2078-2079.

18. McKenna A, Hanna M, Banks E, Sivachenko A, Cibulskis K, Kernytsky A, et al. The Genome Analysis Toolkit: a MapReduce framework for analyzing next-generation DNA sequencing data. Genome Res 2010;20:1297-1303.

19. DePristo MA, Banks E, Poplin R, Garimella KV, Maguire JR, Hartl C, et al. A framework for variation discovery and genotyping using next-generation DNA sequencing data. Nat Genet 2011;43:491-498.

20. Cingolani P, Platts A, Wang LL, Coon M, Nguyen T, Wang L, et 
al. A program for annotating and predicting the effects of single nucleotide polymorphisms, SnpEff: SNPs in the genome of Drosophila melanogaster strain w1118; iso-2; iso-3. Fly (Austin) 2012;6:80-92.

21. Ng PC, Henikoff S. SIFT: predicting amino acid changes that affect protein function. Nucleic Acids Res 2003;31:3812-3814.

22. Smigielski EM, Sirotkin K, Ward M, Sherry ST. dbSNP: a database of single nucleotide polymorphisms. Nucleic Acids Res 2000;28:352-355.

23. Landrum MJ, Lee JM, Riley GR, Jang W, Rubinstein WS, Church DM, et al. ClinVar: public archive of relationships among sequence variation and human phenotype. Nucleic Acids Res 2014;42:D980-D985.

24. Rhead B, Karolchik D, Kuhn RM, Hinrichs AS, Zweig AS, Fujita PA, et al. The UCSC genome browser database: update 2010. Nucleic Acids Res 2010;38:D613-D619.

25. Kananura C, Haug K, Sander T, Runge U, Gu W, Hallmann K, et al. A splice-site mutation in GABRG2 associated with childhood absence epilepsy and febrile convulsions. Arch Neurol 2002;59:1137-1141.

26. Carvalho GA, Weiss RE, Refetoff S. Complete thyroxine-binding globulin (TBG) deficiency produced by a mutation in ac- ceptor splice site causing frameshift and early termination of translation (TBG-Kankakee). J Clin Endocrinol Metab 1998; 83:3604-3608.

27. Parkinson DB, Thakker RV. A donor splice site mutation in the parathyroid hormone gene is associated with autosomal recessive hypoparathyroidism. Nat Genet 1992;1:149-152.

28. Nielsen R, Paul JS, Albrechtsen A, Song YS. Genotype and SNP calling from next-generation sequencing data. Nat Rev Genet 2011;12:443-451.

29. Wendl MC, Wilson RK. Aspects of coverage in medical DNA sequencing. BMC Bioinformatics 2008;9:239.

30. Pan H, He Z, Ling L, Ding Q, Chen L, Zha X, et al. Reproductive factors and breast cancer risk among BRCA1 or BRCA2 mutation carriers: results from ten studies. Cancer Epidemiol 2014;38:1-8.

31. Wooster R, Bignell G, Lancaster J, Swift S, Seal S, Mangion J, et al. Identification of the breast cancer susceptibility gene BRCA2. Nature 1995;378:789-792.

32. Couch FJ, DeShano ML, Blackwood MA, Calzone K, Stopfer J, Campeau L, et al. BRCA1 mutations in women attending clinics that evaluate the risk of breast cancer. $N$ Engl $J$ Med 1997;336:1409-1415. 